\title{
Narrowband THz generation via chirp-and-delay in PPLN
}

\author{
Frederike Ahr ${ }^{1,2}$, Spencer W. Jolly ${ }^{3,4}$, Nicholas H. Matlis ${ }^{1}$, Sergio Carbajo ${ }^{1}$, Koustuban Ravi ${ }^{1,5}$, \\ Tobias Kroh $^{1}$, Jan Schulte ${ }^{1}$, Damian N. Schimpf ${ }^{1}$, Andreas R. Maier ${ }^{4}$, Franz X. Kärtner ${ }^{1,2,5}$
}

\author{
${ }^{1}$ Deutsches Elektronen Synchrotron (DESY) \& Center for Free-Electron Laser Science, Notkestrasse 85, 22607 Hamburg, Germany \\ ${ }^{2}$ Department of Physics, University of Hamburg, Luruper Chaussee 149, 22761 Hamburg, Germany \\ ${ }^{3}$ Institue of Physics of the ASCR, ELI-Beamlines project, Na Slovance 2, 18221 Prague, Czech Republic \\ ${ }^{4}$ Center for Free-Electron Laser Science \& Department of Physics, University of Hamburg, Luruper Chaussee 149, 22761 Hamburg, Germany \\ ${ }^{5}$ Research Laboratory of Electronics, Massachusetts Institute of Technology, Cambridge, MA 02139, USA
}

High-peak-field, narrowband terahertz (THz) sources are well suited for compact electron acceleration [1], however, these sources are still under development and yet to reach optimal energies. A high conversion efficiency via difference frequency generation is established by quasi-phase matching in periodically poled lithium niobate (PPLN). Moreover, to achieve the required $\mathrm{mJ}-$ level THz pulses high energy input pulses are inevitable [2]. Chirpand-delay pumping offers a method to increase the NIR pulse duration and to scale up the energy without damaging the crystal. Here, we present chirp-and-delay in PPLN of varying poling periods and study the effect of crystal length and temperature.

A $5 \mathrm{~Hz}$ Ti:Sa-based laser system (ANGUS) delivers uncompressed pulses up to $5 \mathrm{~J}$ with a $35 \mathrm{~nm}$ FWHM bandwidth at $800 \mathrm{~nm}$ with a pulse duration of $260 \mathrm{ps}$. A train of this chirped pulses with a delay $(\Delta t)$ co-propagates through the PPLN mounted in a cryostat for cooling (Fig. 1a). In order to verify the mechanism we measured the dependence of the THz output on the delay and frequency of the generated THz $\left(\Omega=2 \pi v_{\mathrm{THz}}\right)$, which is related to the poling period as: $\Omega=2 \pi c /\left(\Lambda\left|n(\Omega)-n_{\mathrm{g}}\right|\right)$ [3]. The THz pulse energy was measured with a pyro detector (Fig. 1b) and the THz frequency with an interferometer (Fig. 1c). The THz frequency was varied by using crystals with the poling period of $\Lambda=125,212$, and $400 \mu \mathrm{m}$. Fig. 1d shows that the optimum delay $\left(\Delta t_{\mathrm{opt}}\right)$ depends inversely on the poling period. The substructure for higher delays originates from the third-order dispersion in the chirped pulses and the pulse train. The relation between the measured delays and the measured frequencies agrees well with our prediction using the GDD $\left(\phi_{2}\right)$ of the pulses: $\Delta t_{o p t}=\Omega \phi_{2}$.

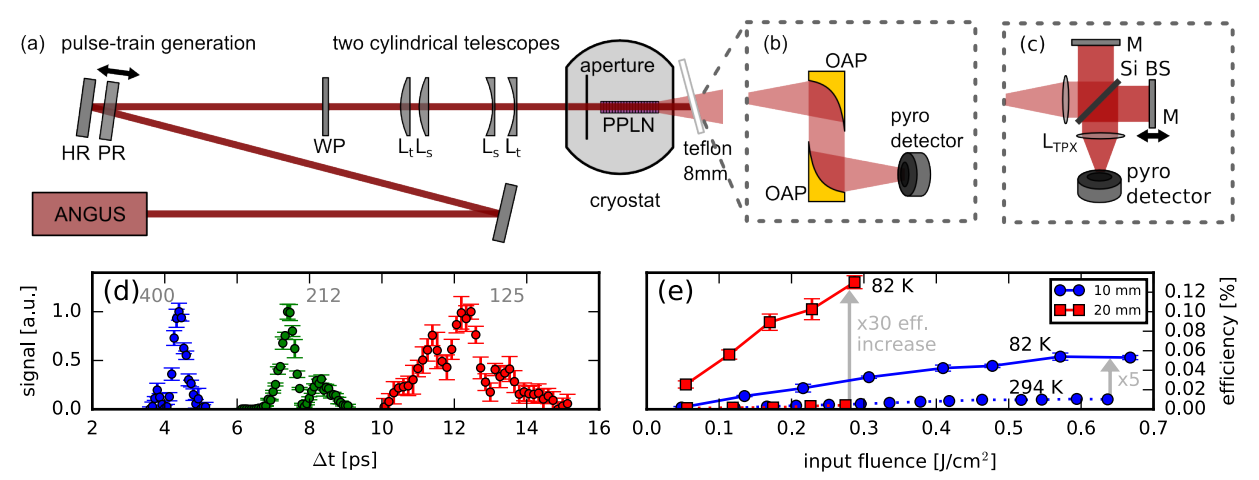

Fig. 1. a) Setup to generate a train of chirped pulses. Detection setup for b) THz pulse energy and c) THz frequency. KEY: PR/HR: partial/high reflector; WP: half wave-plate; $\mathrm{L}_{s / t / T P X}$ : sagittal/tangential/TPX lens; M: Silver mirror; Si BS: silicon beam splitter. d) Normalized THz signal vs. delay for poling periods of 400, 212, and $125 \mu \mathrm{m}$. e) Conversion efficiency of $212 \mu \mathrm{m}$ poling period PPLNs at room- (dotted) and cryogenic (solid) temperature as a function of pump fluence.

Figure 1e shows the internal efficiency vs. pump fluence at room and cryogenic temperatures for two crystal lengths of $212 \mu \mathrm{m}$-poled PPLN. A record extracted multi-cycle THz energy of $40 \mu \mathrm{J}$ was achieved at a frequency of $0.544 \mathrm{THz}$ using the $10 \mathrm{~mm}$ - long crystal $(3 \mathrm{~mm} \times 10 \mathrm{~mm}$ aperture) at $82 \mathrm{~K}$. Although the $20 \mathrm{~mm}$ piece exhibited a higher peak efficiency of $0.13 \%$ already at lower pump fluence, the energy output was less due to a smaller aperture. Optimizing the pump laser bandwidth and increasing the aperture size, chirp-and-delay pumping of PPLN is a promising candidate to reach the mJ-level of multi-cycle $\mathrm{THz}$ pulses with conversion efficiencies on the 1\%level.

\section{References}

1. F. Kärtner et al., "Axsis: Exploring the frontiers in attosecond x-ray science, imaging and spectroscopy," Nuclear Instruments and methods in Physics Research A 829, 24 (2016).

2. K. Ravi, D. N. Schimpf, and F. X. Kärtner, "Pulse sequences for efficient multi-cycle terahertz generation in periodically poled lithium niobate," Opt. Express 24, 25,582 (2016).

3. K. L. Vodopyanov, "Optical generation of narrow-band terahertz packets in periodically-inverted electro-optic crystals: conversion efficiency and optimal laser pulse format," Optics Express 14, 2263 (2006). 